\title{
An Implicit Voice Conferencing System
}

\author{
Markus Töpfer ${ }^{*}$ and Dr. Rolf Kozlowski ${ }^{\dagger}$ \\ German Space Operations Center (GSOC) - German Aerospace Agency (DLR), Weßling, 82234, Germany
}

The Voice Communication Group of the German Space Operations Center started an initiative to abstract voice conferencing systems to an extent that allows the provision of the essential nature and functionality as a coherent whole with minimized technological requirements for the user side. Central intention behind that project is to try to answer three initial questions. How far is it possible to reduce voice conferencing systems to logical functions? What could be done to minimize entry barriers to use the system, as well as how could these logical functions implemented in a modular and still automated and highly flexible way? All, related, embedded and integrated, in the context of operational environments of Space Mission Control Centers. When talking about voice conferencing in Mission Control Centers, the first thing is to clarify the relevance and setup of voice conferencing systems in these environments. Communication is one of the central and key components for operation environments. The most natural way for communication between humans is by voice. Within Mission Control Center environments voice communication is organized as functional group communication. Each communication group uses a specific context for their conversations. This context is defined based on a user role like a Flight Director, or a conversion topic like Water Pump or even a physical channel with unique characteristics like VHF1. The context is explicit, predefined and called a Voiceloop. The definition is done outside of the voice conferencing system, whereas the context in use is dependent to the users current operational communication needs and fully controlled by the individual user. For the system design, these two boundaries for system control were the basement and start of the definition. A flexible input for setup of communication groups, as well as a flexible input of actual participation control. Actual communication capabilities are setup in a fully automated way, dependent on these inputs. The control interfaces are implemented using a web based approach with Representational State Transfer. This way the interface stays highly flexible for implementation or integration within different architectures. The prototype provides all needed functionalities over a website. As prerequisites for the user side a current up to date web-browser and some kind of audio input and output device like a headset, as well as a network connection to the core system are required. There is no special device, no dedicated keyset and no separated network needed. For the administration side a commonly used standard user repository was used, OpenLDAP. Lightweight Directory Access Protocol (LDAP) is a protocol to organize and maintain distributed directory information services. Within the LDAP server a new scheme was implemented to manage contexts of communication groups (voiceloops). Starting from this structure the whole access permission set will be generated and distributed automatically. It is made accessible by Unified Resource Locators (URL), the common addressing scheme of web based structures. The URL scheme is unified for each individual user and stored as a unique webpage dedicated to the user. This webpage is offering his control interface for communication context selection, after successful authentication at the webserver. Audio distribution is performed over a communication protocol implemented within current web browsers. WebRTC is defined by the W3 consortium, the web standards committee, as Web Real Time Communication. It is a capability to access local media streams of audio and video devices of a PC, Tablet or Phone directly from a website through the web browser. It is programmable by some JavaScript Application Programming

\footnotetext{
* Voice Systems Engineer, DLR RB-KOB Communications and Ground Stations, Münchener Straße 2082234 Weßling Germany, Markus.Toepfer@DLR.DE

${ }^{\dagger}$ Departement Head, DLR RB-KOB Communications and Ground Stations, Münchener Straße 2082234 Weßling Germany, Rolf.Kozlowski@DLR.DE
} 


\begin{abstract}
Interfaces (APIs). Beside the access to local media streams it is used to distribute the media over remote media streams to different web browsers. The system idea presented in this paper is reduced to the core functionality of manual predefinition of communication groups and permission sets by administration, combined with automated deployment and permission enforcement, while providing full participation control to the end user, independent of his location. The approach was to start from scratch for Mission Control Room Conferencing, and is a feasibility study based on state of the art web technology.
\end{abstract}

\title{
I. Introduction
}

$\mathrm{T}$ HIS paper is discussing the topic Voice Communication Systems in Space Mission Control Center environments. Voice Communication Systems are major Ground Segment subsystems, which provide intercommunication between ground, astronauts onboard space vehicles and various distributed ground centers, which may be spread internationally based on specific mission characteristics.

Human communication in Mission Control Room environments is of a formal nature with well-structured and predefined communication groups. Main intention behind voice communication for mission control is to enable information exchange for decision making processes and to command and organize team activities.

The main differentiating characteristic to common conferencing solutions is the provision of multiparty multiconferencing capabilities. In a multiparty conference several interested parties are connected over some kind of technology which transmits all incoming traffic to all of the participants. That is the classical conferencing scenario, which creates a loop over all of the connections. In a multiconferencing scenario each participant is able to join several multiparty conferences at the same time.

In Space Mission environments the term Voiceloop is used to identify a specific predefined multiparty conference. This conference is defined by external authorities, which identify a demand for a dedicated Voiceloop for their specific mission needs. Within the definition it is predefined where the Voiceloop needs to be present (location) as well as who has access to that Vocieloop. Access itself may be restricted to listen only or as talk enabled access for a participant. A communication participant may be defined over a role (e.g. Flight Director) or a dedicated user. To simplify access restrictions and permissions for operational use cases of 24/7 shift scenarios, where a position like Flight Director is occupied by several individual users, Role Based Access Control is most commonly used.

Voice Communication Systems get this predefinition as an input and must provide the communication capabilities. Therefore they need to connect all involved locations, enforce the rules for access control and provide each user selection and participation capabilities for multiple conferences at the same time.

Traditionally the systems are built dedicated based on the mission requirements. Required ground centers are connected, communication paths are set up and dedicated voice communication equipment is installed at the various locations. For center to center connections available communication paths are adapted and refactored to changed needs. Another strategy for more lightweight connection requirements are PABX calls between the different locations and implementation of access control by Caller ID checks and manual or automated patches to the Voiceloops.

Taking into account the support of multiple and changing mission needs of a Control Center time, effort and costs required to setup and implement a Voice Communication System are very high and flexibility in the setup is limited. At the same time Space Missions are currently opening up to a much wider audience. Universities, private companies and other research institutions show interest in experimenting in space. Smaller entities of Space Agencies increase their activities and the user base for Voice Communication Systems connected and involved in Mission Control Room communication is growing beyond the standard support of flying spacecrafts.

Usually these kinds of missions do have a limited amount of money to spend for infrastructure and moreover try to spend as much as possible for their mission targets. They are also interested in any communication related to their missions, be it related to scheduling, conduction, possible results or problems. They even may be located at different locations in itself, for example based on an international cooperation between universities. Examples for these kinds of missions are experiments on board of a space station, small research satellites or within other community like environments related to space missions like for example PlanetLabs.

To support of these kind of activities integrated in the overall operational concept of Space Mission Operations out of the shelf systems does not exist. Lightweight and virtualized voice communication systems for Mission Control Room environments, which support operational prerequisites in form of predefined communication groups, 
as well as use cases involving non dedicated Thin Clients supporting multiconferencing scenarios, are also not available.

Within this paper we will show a potential conceptual approach, which abstracts Voice Communication Systems to logical functions. The system itself will work over any IP based network.

Following conditions for the system are taken into account:

1) Communication groups are predefined using Role Based Access Control within LDAP

2) A participant has access to a device with an up to date web browser and a connected headset

3) The user device has network access (to a system termination endpoint)

4) System termination endpoints are interconnected

5) System terminttion endpoints are connected to the LDAP repository hosting the configuration

6) Outgoing network traffic is not blocked by means of packet filter implementations

We will present a concept which shows that it is possible to reduce the physical system setup to an extent, which allows a full automated provision of communication capabilities and access control functions using a standard web browser and headset at client side out of an operational input; the definition of communication groups.

\section{User Side of the System}

The user side of the system consists of two main functionalities. Provision of an interface for the selection of required Voiceloops to support the current task of a user and actual provision of voice media streams of all selected Voiceloops. Figure 1 Example User Interface shows a prototype of a control interface developed based on web technologies during the conception phase. A user $\log$ in on a web page and selects his role in use. Control for all Voiceloops assigned to that role will be made available within a HTML5 based interface.

A user can select the participation state for each loop individually and select between listen only or talk enabled participation. Each Voiceloop has individual volume controls for loop based fine control of audio streams. Talk selected Voiceloops are shown with a green border in this prototype, listen only selected loops are shown with a blue border.

This development version of a potential interface has 2 main areas, one to control Voiceloops and a general control area with 3 buttons for interactions.

Selection of Voiceloops is implemented with toggle buttons, which switch between listen only and

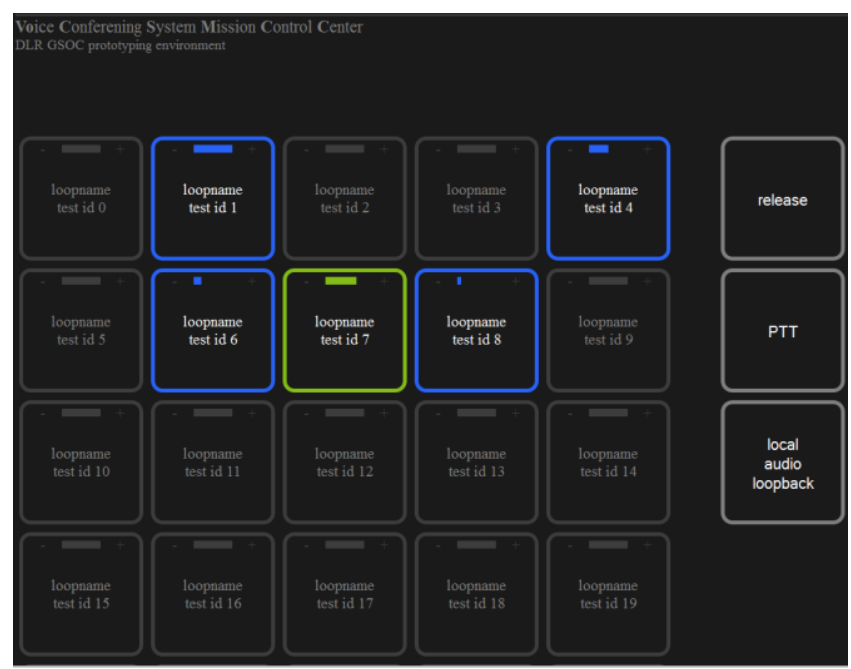

Figure 1 Example User Interface talk enabled as long as a Voiceloop was selected. The PTT button is used to open the microphone. A local audio loopback button provides a test environment to check the local audio setup. The release button is used to deselect an enabled conference. (Switch off toggling between monitor and talk)

Voice media streams are connected using an emerging technology, Web Realtime Communication (WebRTC) ${ }^{1}$. WebRTC enables on one hand access to local media devices like microphone, speaker and camera internally within a web browser and on the other had to connect these devices to remote media streams and vice versa. This technology enables the provision of multiparty conferencing environments with centralized stream multiplication. Another emerging technology in the HTML5 world is WebAudio ${ }^{2}$. WebAudio is defining an API for mixing of different remote audio streams within a web browser. A combination of these 2 APIs will allow the provision of Multiparty Muliconferencing clients over a simple web page using HTML5 technology.

First functional tests are very promising, even if both specifications are not yet in a final accepted state. However Mozilla's Firefox, Google's Chrome and Opera already support the APIs enabled by default in their current stable versions".

\footnotetext{
$\$$ http://www.webrtc.org/ visited 04.04.2014
} 


\section{Administration Side of the System}

For administration and setup of the permission and access rules a standard LDAP ${ }^{3,4}$ environment is foreseen. Lightweight Directory Access Protocol (LDAP) is specified as open internet standard to organize directory structures.

Voice Communication Systems use a Role Based Access Control schema, where users are assigned to roles and roles are assigned to Voiceloops with the respective access permission of talk or listen only. Such a schema enables the use of templates for roles, and user management is much simpler and better organized. LDAP may be extended with application specific needs using a customized LDAP schema ${ }^{5}$.

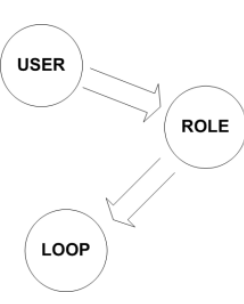

Figure 2 RBAC

To enable the permission handling for Voice Communication Systems a dedicated schema was created. It defines the Objectclass Voiceloop within a standard LDAP and extends that class with the 3 attributes VoiceloopName, VoiceloopMemberTalk and VoiceloopMemberListen. This schema is written in ASN. ${ }^{6}$ syntax like shown in Figure 3 Voice communication specific schema extension for LDAP. This file contains everything to manage Voiceloops with Role Based Access Control assignments within LDAP over the directory information tree. The unique object identifier (OID) with number 41227 was requested at the Internet Assigned Numbers Authority (IANA) $)^{\S}$ for the project vocsmcc (Voice Communication

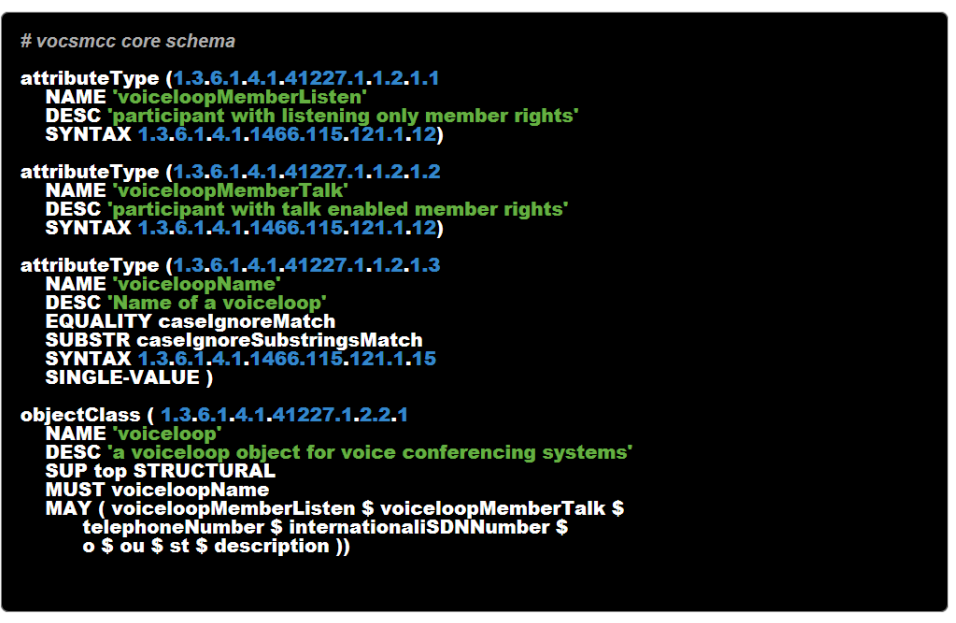

Figure 3 Voice communication specific schema extension for LDAP Systems Mission Control Center).

\section{High Level System Concept}

The basic high level idea of the concept is to set up an environment using a LDAP server, where all of the group communication definitions are stored, managed and distributed. This server may be dedicated to the Voice Communication System or shared as an integrated server for overall operational permission handling.

Control and Media plane for user interactions are separated functions implemented with web pages and media relay functionality of termination endpoints. The whole system is on top of the network layer of the OSI model ${ }^{7}$.

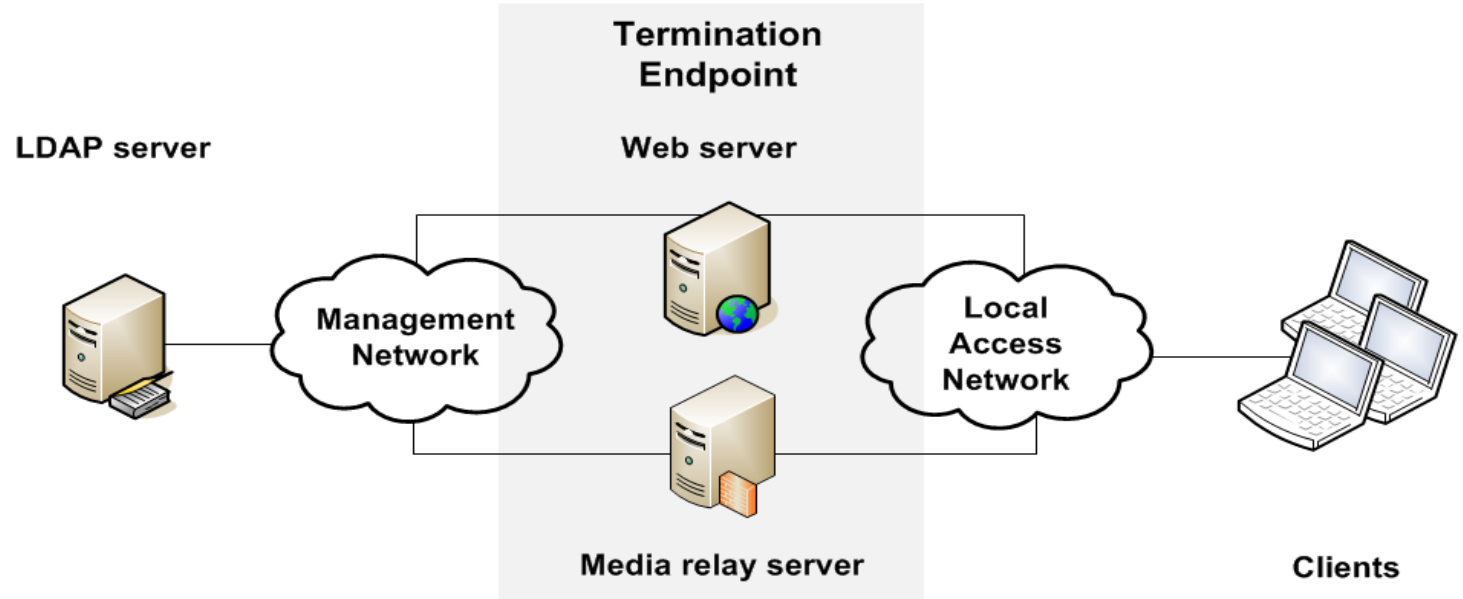

Figure 4 General system functionality

${ }^{\S}$ https://www.iana.org/

American Institute of Aeronautics and Astronautics 
On a client in the access network of a termination endpoint a web browser is used to connect to the webserver of the termination endpoint. After user authentication and selection of the desired role the user interface is presented. Within the user interface all loops related to the role of the user are shown and interaction to connect to the loops is available. To connect to any loop the user presses or clicks on a button and the connection to the media relay will be established. The media relay is performing all actions to route voice traffic to any connected participant.

In case of a new operational concept, like new roles, assignment of a new user, a new mission with new loops, or the extension of a user role with a new loop the LDAP definition will be updated. As soon as an update is available the concept foresees a regeneration of the webpages, the user interface for each user. At the same time new loops will be made available at the relay server for the provision of voice routing.

All of these steps to rewrite and update user interface, media relay connections and even system interconnections shall be performed fully automatically including consistency checkouts and automated internal tests. This implies that extension of a Voice Communication System with new mission parameters will be done fully automated, may be done on the fly and even without any human interaction for system configuration. Each change shall be stored in a repository for immediate manual rollback to the previous version.

\section{Network Concept}

Connections between (Web) Client and termination endpoints, as well as interconnections between termination endpoints shall be IP based connections. Physical connections may be IP over Ethernet, IP over WLAN, IP over LTE or even IP over HDMI. For the time being no physical connection method is preferred and the actual physical connection is not part of the concept proposal and will be defined and analyzed in a following study period.

The network concept is aligned to the requirements for WebRTC based systems. WebRTC is specified to connect Web browsers for realtime communication. The connection will not be done with media links over the web server, instead direct media links between $2 \mathrm{Web}$ browsers are foreseen. To identify the counterpart for media termination Internet Connectivity Establishment (ICE) ${ }^{8}$ is used. ICE makes use of Session Traversal Utilities for NAT (STUN) ${ }^{9}$ and Traversal Using Relays around NAT (TURN) ${ }^{10}$.

For each of the used techniques a public available relay server needs to be accessible to establish a media connection. For STUN based connections both Clients $\mathrm{A}$ and $\mathrm{B}$ will request the respective Public IP addresses from the STUN server, and need to exchange this information between each other. Afterwards the media stream will be established using the Public IP of Client A and the Public IP of Client B.

TURN is a relay technique on top of STUN, which terminates media connections using the Public IP of Client A connected with a media stream to the Public IP of the relay server bridged within the relay server to the media stream of Public IP B.

Both technologies where developed to support clients behind NAT and firewall enabled networks to connect to each other without previous knowledge of the counterparts IP address information.

With these technologies all information to connect between each other are made available from the relay server. Address of the relay server will be transmitted within the Webpage for the client interface. If relay and web server

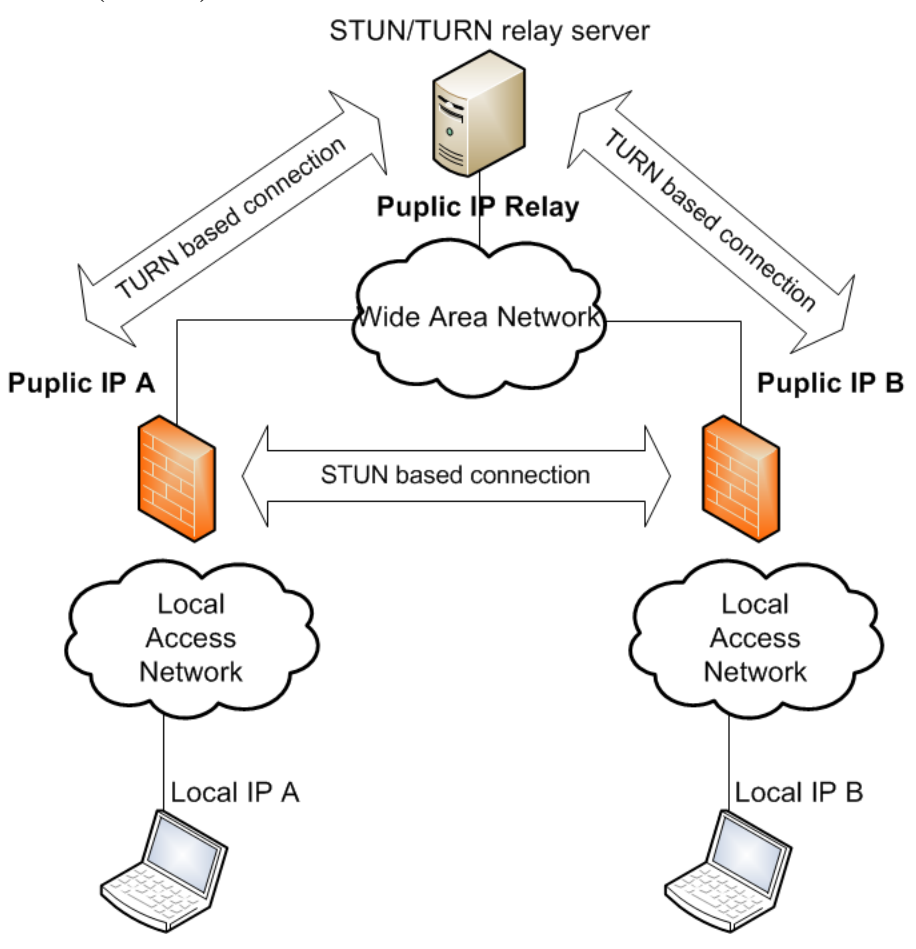

Figure 5 Overview STUN/TURN are running together on a termination endpoint 2 ports and one IP address need to be available for Client connections. Port 80 for website access and Port 3389 to access the STUN/TURN relay. In addition to this one more network functionality is required. It must be available that opening ports for outgoing connections is on demand in the network of the clients. 


\section{A. Session Traversal Utilities for NAT (STUN)}

Functionality of STUN is used from clients to request the Public IP and port for their own connection to the STUN server. A message is sent from LocalIPClient:RandomPort over PublicIPClient:RandomPort to PublicIP:3389 of the STUN server and a replay including PublicIPClient:RandomPort is sent back. The tuple PublicIPClient:RandomPort will be used for direct connection establishment between different clients. Exchange of this information between clients is out of the STUN specification and need to be done separately.

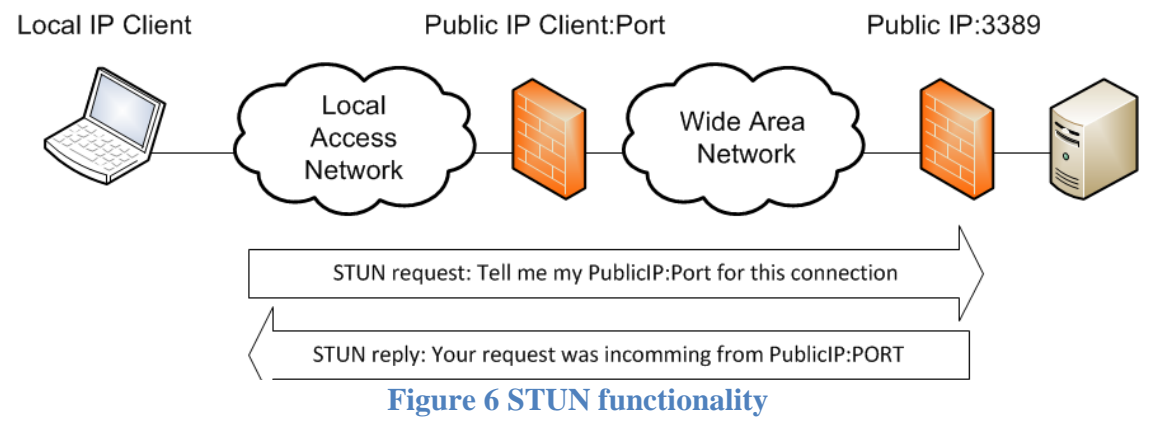

After exchange of the information of the tuple with another client the media connection can be established directly between the Public IP's of the clients. STUN works with or without authentication methods.

\section{B. Traversal Using Relays around NAT (TURN)}

TURN is an extension of STUN and provides the same functionality like STUN if a STUN request is sent to the same server. If the request is identified as TURN, the server starts an internal process to open up a new process for connection establishment with a PublicIP_TURNserver:RandomPort. This address is sent back to the client and the client connection is attached to the process on the new opened port. This way connections are bridged over a TURN relay server.

\section{Session establishment}

Communication sessions are always initiated from the secured local environment of the client. A website request is sent from the client to a termination endpoint on port 80 . The reply with the website contains the information for the relay functionality. This functionality may be running on the same IP like the webserver or on a different one. However the media connection setup is also always established from the secured environment to the relay server on port 3389.

TURN and STUN based environments may be cascaded, which is part of the configuration for the relay functionalities. With cascaded relays sessions may be established over several separated network areas.

Media description exchange in form of Session Description Protocol (SDP) ${ }^{11}$ messages are exchanged over the established connection link with STUN/TURN between different clients to identify:

1. Type of media

2. Transport protocol

3. Format of media

4. Remote address for media

5. Remote transport for media

Remote address and remote transport are the parameters set by the output of the STUN/TURN methods. Type, transport protocol, and format may be pre specified by configuration especially for conferencing environments.

\section{Extensions for Media Relay functionalities for conferencing scenarios}

The TURN specification identifies the connection of 2 media streams over a relay server. It enables communication between 2 web applications. With this technology an environment for direct media links between all clients may be established, but this technique is not able to support conferencing out of the shelf. No multiplication of media streams is specified, which means for a conference all clients connected to that conference need to establish dedicated media streams between all other connected clients.

TURN works will allocations. An authenticated client A allocates a new session, which creates a new connection possibility on the TURN server on PublicIP_TURNserver:RandomPort. Another client B will connect to this tuple to establish a media link to the initiating client. Both connections to the server ClientA:PortA to PublicIP_TURNserver:RandomPort and ClientB:PortB to PublicIP_TURNserver:RandomPort are bridged within the TURN relay. This means every incoming message is simply relayed to the other connection participant.

For conferencing scenarios the TURN relay functionality may be extended with a stream multiplication functionality. If type, transport and format of media are pre specified by configuration all clients will use the same 
formats and media types. Stream multiplication may be done in such cases by simply copying of the whole package and streaming these packages to all interested clients. Therefore each allocation for a Voiceloop needs to be done by configuration. As an example Voiceloop "Test1" is assigned to run on port 10000. An allocation on the TURN relay is done to enable connections to port 10000 for authenticated clients. If client A requests participation to the Voiceloop it will establish a connection to PublicIP_TURNserver:10000 instead of opening up a new allocation. The same will be done by client B,C and so on. Each client connects to port 10000 at the relay. All incoming media messages on port 10000 will be transferred to all connected clients.

This extension will work within any web browser. This way a Voiceloop may be available by configuration of a relay node.

The extension of TURN works with multiple unicast streams and stream multiplication within a termination endpoint. Configuring the Voiceloop "Test 1 " on port 10000 for all connected termination endpoints will provide conferencing functionalities for this Voiceloop over all termination endpoints.

\section{E. Statement Network approach}

The described functionality in the previous paragraph is based on multiple unicast connections. Instead of direct multicasting this functionality works out of the box for standard web browsers without any plugins. In addition it does not require connections of multicast enabled networks and specific multicast bridges between network segments. A multicast based solution for a similar functionality is described in "Prototyping an Event based Distribution Model for Multicast based Multiconferencing with SIP",12.

Locating the termination endpoints close to clients e.g. by connecting them to the same switches will have very small overhead in relation to a multicast based solution, but reduce network complexity and implementation requirements for the underlying topology significant. At the same time the connection is always under full control for e.g. switching to a different termination endpoint in case of maintenance or failure investigation activities at the application layer instead of the network layer.

The approach behind this topological choice is at one hand the direct support for WebRTC streams and on the other hand implementation possibilities for Software Defined Network ${ }^{13}$ like solutions on top of the network layer, which makes the whole system more independent from the used network. This solution is split into a Control Plane (over a webpage) and Media Plane (over TURN relays) for user based operations.

With this solution all logic for connections, failover strategy's and network connections is done within the application itself. It enables the connection of network segments, which are not under control of the network department of the Mission Control Center, which provides the Voice Communication System capabilities.

\section{F. System setup}

For the system an external available standard STUN/TURN media relay server need to be available to implement the functionality for unknown client connections. This server also needs to host the initial website for authentication of the user. The webpage may be as simple as possible with a redirect to the termination endpoint of network A. This way all access information and authentication will be done within the controlled environment of A.

The public available server may be accessible using the domain https://vocsmcc.com. It will redirect to the domain https://voice.gsoc.dlr.de. The user will authenticate at the GSOC domain to request the user interface containing the information for media connections. Media connections will be established using STUN://vocsmcc.com:3389. This connection will open a direct link between the termination endpoint within network $\mathrm{A}$ and the client

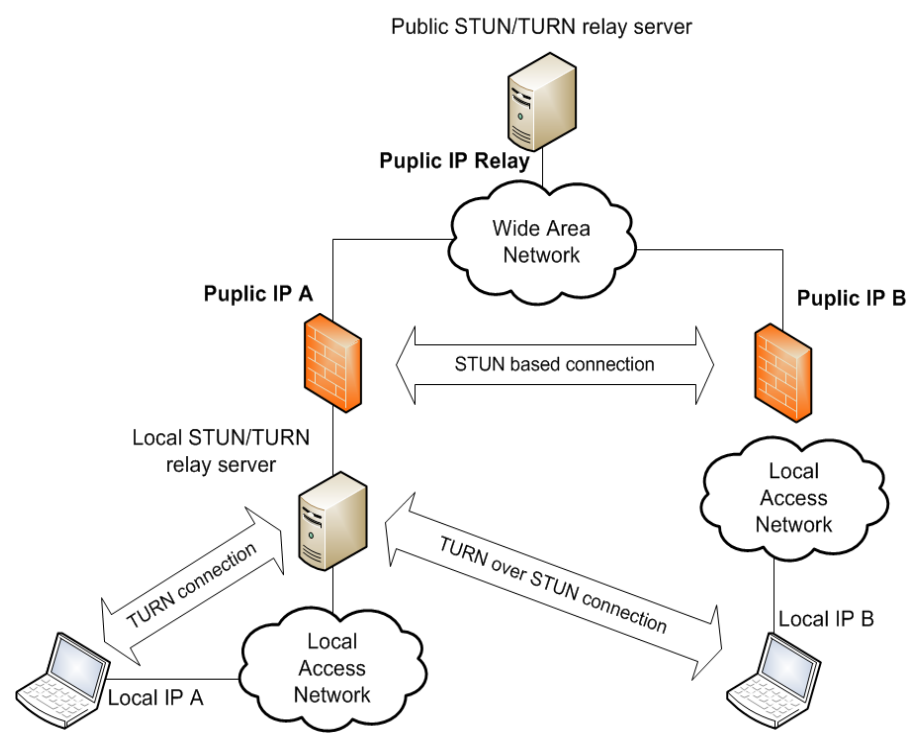

Figure 7 External Client to Mission Control Voice Communication connected in network B. This way the client is able to connect to the conferences available at TURN://voice.gsoc.dlr.de over a random port number. By selecting the Voiceloops over his interface he will request 
the preconfigured allocations for the conferences at the server of side $\mathrm{A}$ and be able to listen to the Voiceloops present at termination endpoint A. This technique combines TURN over STUN connections with multiple unicast media relaying at the termination endpoint in network A. At the same time the combination of STUN and TURN will prevent open ports for connections to the local termination endpoint in network A, as all requests will be initialized from the termination relay over STUN to the client in network B. Port's at the firewall will be enabled outgoing on demand for connections specifically to client B. For each Voiceloop selected at client B a dedicated media connection will be set up with such kind of solution. The media path is direct between the Public IP addresses of the network locations $\mathrm{A}$ and $\mathrm{B}$ without an additional external media relay.

To interconnect dedicated systems at different sides an implementation for the exchange of configuration information needs to be specified, which is out of scope for the current phase of the conceptual design, but will be done in a later phase of the architecture definition.

Instead of public connections over the Internet local MPLS paths may be used with the same kind of setup and technology, but this would limit possible connections to existing MPLS trunks, which is out of scope for the initial intention to open up the system for simple access of external entities.

The relay may be implemented on e.g. the network side A to enable external connections directly to the URI of location A but is needed for automated transmission of configuration data between interconnected sides for hub to hub based implementations using auto recovery. This technic is part of the overall conceptual design but not described within this specific paper.

\section{System Description}

Elements of the system are LDAP servers, termination endpoints and web browsers connected over network links. External network connections between a requester of system capabilities and a termination endpoint are required, but not a direct part of the system itself. Clients are virtual and instantiate at system access on the fly based on web applications.

A system configuration for access permissions is stored within a LDAP repository. This repository may be requested automatically in periodical intervals to check for configuration changes, or manually on request, dependent on the operational requirements. For each user with voiceloop access dedicated web page information are created as static websites on the web server component of the termination endpoint. These web pages contain the user interfaces for all of the roles a user is assigned to. In addition to the webpages JavaScript files are used for interaction definition. These scripts contain the functionalities for media setup, checks and transmission.

Users connect to the system at a specified and pre communicated URI ${ }^{14}$. This may be for example https://voice.gsoc.dlr.de. The initial web page will be a general one containing a login area. At the login area the user uses his credentials and after successful verification he will be redirected to his entry page at https://voice.gsoc.dlr.de/user. This page will list all of the roles configured for this user. After selection of the role fitting his current operational tasks (e.g. role Flight Director or role Simulation Flight Director) he will be forwarded to the page https://voice.gsoc.dlr.de/user/role. This pages contains the user interface for interactions with Voiceloops configured for this role like shown in Figure 1 Example User Interface.

All of these information are automatically generated out of LDAP by a reverse search of the objectclass Voiceloop. In addition the system is already multiple LDAP capable and checks for homonymy at all URI levels (user, role, voiceloop)

To connect to a voiceloop a request is send to $h$ ttps://voice.gsoc.dlr.de/user/role/voiceloop. The web server replies with the IP and port number assigned for that Voiceloop and this user at that moment. This technique is used to scale out users to different media termination endpoints in case of higher system loads. It is a dynamic assignment of media relay endpoints.

Using static web pages and URI schema with everything below a user account will give the possibility to limit access at file system level of the web server to the requesting user. This way out of the box file system security of Linux will be used for user interactions. (Files below https://gsoc.dlr.de/user are access restricted to user:user)

The media component of the termination endpoint can be preconfigured out of LDAP too, by extending the base schema for vocsmcc with an additional attribute: voiceloopPortNumber. Assigning port numbers to Voiceloops within LDAP will currently break the multiple LDAP functionality and therefore a redefinition is under investigation. Nonetheless the conceptual approach is to hold this configuration at the LDAP server too. For the time being the port number to access the media component of the termination endpoint is included within the script generation functionality.

If a user selects a voiceloop for participation a media stream to the termination endpoint is opened after the server replies with the IP and port to be used. Each voiceloop connection will have a dedicated media stream 
between termination endpoint and web client. Mixing of these streams is done within the web browser using the WebAudio API.

The whole system of web pages and related JavaScript files is automatically written to static web pages. This way the whole setup is testable with automated checkout functions and any configuration failure can be excluded at voice System level. If the LDAP repository holds a valid configuration for the mission the voice system will hold a valid configuration too.

\section{Conclusion}

The concept description of this paper shows a possible solution for a lightweight, fully automated and implicit Voice Conferencing System using virtual clients which instantiate based on HTML5 technology within a web browser. It contains highly scalable techniques to enable a huge user base. The system is created out of a configuration repository, which reduces configuration management to zero as it is already the basement for the whole system. Every automated change is stored in a repository for fast rollback scenarios and contains checkout functionalities for integrated testing.

All technology used for system setup and functionality is widely used within the Internet and therewith tested at the largest possible scale.

The system is independent form the networks used and may be implemented on top of wireless or wired networks, public or private networks or even dedicated network trunks for voice communication systems. It may be implemented as the lightweight solution shown in this concept or within an adapted more dedicated version.

Entry barrier to access the system are minimized to web browser based access with devices containing microphones and speaker systems, which are available on nearly any notebook, tablet or smartphone sold in the last years.

The standard web technology TURN is extended with a media relay functionality to implement a multiparty conferencing environment based on predefined media parameter. This functionality will be made available for the public as an extension RFC to the TURN RFC as soon as functional testing is performed for validation of the extension over a full featured prototype implementation.

Multiconferencing functionality is implemented within the web clients. A performance analysis need to be done once an integrated prototype is available.

Connections to existing systems may be done with media gateways connected to the media relays of the termination endpoint. For RTP media streams gateways to any other common media connection are industrial available at large scale from all kind of vendors.

This paper was written as basement for discussions especially at the network layer of the proposed concept as this part is seen as the most unpleasant and revolutionary one when taking into account classical network infrastructure setups for mission control room environments. 


\section{References}

${ }^{1}$ Bergkvist, A., Burnett, D.C., Jennings, C., and Narayanan, A., "WebRTC: Real-time Communication Between Browsers", W3C Editor's Draft [online specification], Version 1.0, URL: http://dev.w3.org/2011/webrtc/editor/webrtc.html [cited 25 March 2014].

${ }^{2}$ Rogers, C., "Web Audio API", W3C Editor's Draft [online specification], Version 1.0, URL: https://dvcs.w3.org/hg/audio/rawfile/tip/webaudio/specification.html [cited 25 March 2014].

${ }^{3}$ Wahl,M., Howes,T., Kille,S., "Lightweight Directory Access Protocol (v3)”, Internet Engineering Task Force RFC [online specification RFC2251], Version 3.0, URL: http://tools.ietf.org/html/rfc2251 [cited 01 March 2014].

${ }^{4}$ Sermersheim,E., "Lightweight Directory Access Protocol (LDAP): The Protocol”, Internet Engineering Task Force RFC [online specification RFC4511], Version 1.0, URL: http://tools.ietf.org/html/rfc4511 [cited 01 March 2014].

${ }^{5}$ Sciberras,E., "Lightweight Directory Access Protocol (LDAP): Schema for User Applications", Internet Engineering Task Force RFC [online specification RFC4519], Version 1.0, URL: http://tools.ietf.org/html/rfc4519 [cited 01 March 2014].

${ }^{6}$ ITU-T study group, “Abstract Syntax Notation One (ASN.1): Specification of basic notation”, ITU-T standard [X.680], Version 1.0, URL: http://www.itu.int/rec/T-REC-X.680-200811-I/en [cited 01 March 2014].

${ }^{7}$ ITU-T study group, “Open Systems Interconnection - Basic Reference Model: The Basic Model”, ITU-T standard [X.200], Version 1.0, URL: http://www.itu.int/rec/dologin_pub.asp?lang=e\&id=T-REC-X.200-199407-I!!PDF-E\&type=items [cited 01 March 2014].

${ }^{8}$ Rosenberg,J., "Internet Connectivity Establishment (ICE) A Protocol for Network Address Translator (NAT) Traversal for Offer/Answer Protocols", Internet Engineering Task Force RFC [online specification RFC5245], Version 1.0, URL: http://tools.ietf.org/html/rfc5245 [cited 01 March 2014].

${ }^{9}$ Rosenberg,J.,Mahy,R., Wing,D. “Session Traversal Utilities for NAT (STUN)”, Internet Engineering Task Force RFC [online specification RFC5389], Version 1.0, URL: http://tools.ietf.org/html/rfc5389 [cited 01 March 2014].

${ }^{10}$ Mahy,R., Matthews,P., Rosenberg,J. “Traversal Using Relays around NAT (TURN): Relay Extensions to Session Traversal Utilities for NAT (STUN)”, Internet Engineering Task Force RFC [online specification RFC5766], Version 1.0, URL: http://tools.ietf.org/html/rfc5766 [cited 01 March 2014].

${ }^{11}$ Handley,M., Jacobson,V., Perkins,C. “SDP: Session Description Protocol”, Internet Engineering Task Force RFC [online specification RFC4566], Version 1.0, URL: http://tools.ietf.org/html/rfc4566 [cited 01 March 2014].

${ }^{12}$ Töpfer,M. "Prototyping an Event based Distribution Model for Multicast based Multiconferencing Parameter with SIP", Thesis, Departement of Electrical Engineering and Computer Science, University of Applied Sciences Bremen., Bremen, 2009.

${ }^{13}$ Open Network Foundation "Software-Defined Networking: The New Norm for Networks", [Whitepaper], Version 1.0, URL: https://www.opennetworking.org/images/stories/downloads/sdn-resources/white-papers/wp-sdn-newnorm.pdf [cited 01 March 2014].

${ }^{14}$ Berners-Lee,T. Fielding,R.,Masinter,L.“Uniform Resource Identifier (URI): Generic Syntax”, Internet Engineering Task Force RFC [online specification RFC3986], Version 1.0, URL: http://tools.ietf.org/html/rfc3986 [cited 01 March 2014]. 